\title{
Electrical control of the light absorption in quantum-well functionalized junctions between thin metallic films
}

\author{
Dana Codruta Marinica, ${ }^{1, *}$ Andrey K. Kazansky, ${ }^{2,3}$ and Andrei G. Borisov ${ }^{1}$ \\ ${ }^{1}$ Institut des Sciences Moléculaires d'Orsay (ISMO), UMR 8214, CNRS, Université Paris Sud, \\ Université Paris-Saclay, bât 520, F-91405 Orsay, France \\ ${ }^{2}$ Material Physics Center CSIC-UPV/EHU, Donostia International Physics Center DIPC, \\ Paseo Manuel de Lardizabal 5 20018, Donostia-San Sebastián, Spain \\ ${ }^{3}$ IKERBASQUE, Basque Foundation for Science, 48013 Bilbao, Spain
}

(Received 5 September 2017; revised manuscript received 22 November 2017; published 11 December 2017)

\begin{abstract}
We use a time-dependent density functional theory approach to study the optical response of a hybrid nanostructure where the junction between thin metallic films is functionalized with a quantum well (QW) structure. We show that an unoccupied QW-localized electronic state opens the possibility of the active electrical control of the photoassisted electron transport through the junction and of the absorption at optical frequencies. Control strategies based on an applied bias or an external $\mathrm{THz}$ field are demonstrated.
\end{abstract}

DOI: 10.1103/PhysRevB.96.245407

\section{INTRODUCTION}

The development of actively controlled optical devices allowing to manipulate light at dimensions well below its wavelength is of paramount importance for various applications such as information transfer, solar energy harvesting, sensing, and many others. Recent advances in active metamaterials [1-4], in active tuning of the plasmon excitations at metal surfaces and in metal nanoparticles [4-13], or in electrically controllable linear and nonlinear optical and plasmonic in-plane response of graphene nanostructures [14-18] provide a few examples of the active research activity in nanoscale optoelectronics. In this respect, photon-assisted electron transport (PAT) $[19,20]$ in the systems where both continuum and discrete electronic states are present opens new appealing perspectives owing to the incident electromagnetic field induced transitions at well-resolved frequencies [21,22]. So far, the PAT in nanostructures with discrete state-continuum coupling such as semiconductor heterostructures [terahertz (THz) frequency range], quantum dots, or metal-molecule interfaces has been mainly described within the one-electron scattering theory framework using the model Hamiltonian techniques [21-34]. Such an approach gives a transparent qualitative insight, but it does not allow one to capture manyelectron effects such as the dynamical screening. In particular, for metals with high conduction electron density, the optical response of the system is determined by the nonlocal response and dynamical screening. Only few studies of PAT based on the time dependent density functional theory (TDDFT) and incorporating many-body effects at full-quantum level have been reported so far [35-40]. However, to the best of our knowledge, the issue of active control has not yet been addressed.

Here, using real-time TDDFT calculations, we follow the electron dynamics triggered by an incident electromagnetic wave in a metal-quantum well-metal sandwich nanostructure. Our results demonstrate that an active electrical control of the optical response of the system can be realized by using

\footnotetext{
*dana-codruta.marinica@u-psud.fr
}

a gateway electronic state of a quantum well (QW) structure located in the junction between thin metallic films. At optical frequencies, the tunneling current across the junction is dominated by the PAT involving the unoccupied QW-localized electronic state. The energy of the latter with respect to the Fermi levels of the leads and thus the ac conductivity of the junction can be actively tuned by (i) using a gate electrode, (ii) applying an external bias across the junction, or (iii) subjecting the junction to a slowly varying $\mathrm{THz}$ electric field. In turn, this allows for active modulation of the optical absorption of the system as we demonstrate below.

The paper is organized as follows. In Sec. II, we describe the studied system and the TDDFT method used. Section III presents the results and their discussion. Section IV provides summary and conclusions. Finally, details on the numerical implementation of the TDDFT procedure are given in Appendix. Atomic units are used throughout the paper unless otherwise stated.

\section{SYSTEM AND METHOD}

The planar metal-QW-metal structure studied in this work is schematically shown in Fig. 1(a). We use the coordinate system with $z$ axis perpendicular to the interfaces with $z=0$ set at the middle of the junction. The system is translationally invariant in the $x$ and $y$ directions. The incident electromagnetic field is linearly polarized along the $z$ axis. The junction consists of two thin metal slabs separated by a narrow gap that can be filled with either an organic self-organized molecular layer [41-44], a graphene layer [45-47], or a metallic QW formed by a metal film of several monolayers width [48,49] isolated from the leads by a dielectric. The electronic structure of the $\mathrm{QW}$ is such that the unoccupied QW-localized electronic state (QWS) is within the $\mathrm{eV}$ energy range as measured from the Fermi level of the slabs, and the HOMO-LUMO gap is wide enough so that the optical excitations from HOMO can be neglected within the frequency range of interest.

In what follows we consider the case of a metallic QW formed by a metal film of several monolayers width sandwiched between thicker metal slabs of width $L=5.5$ or 
(b)

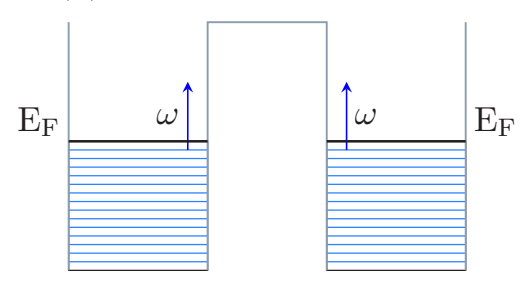

(d)

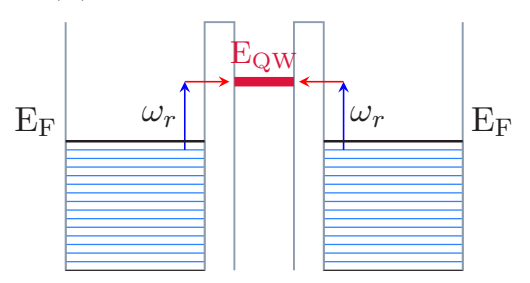

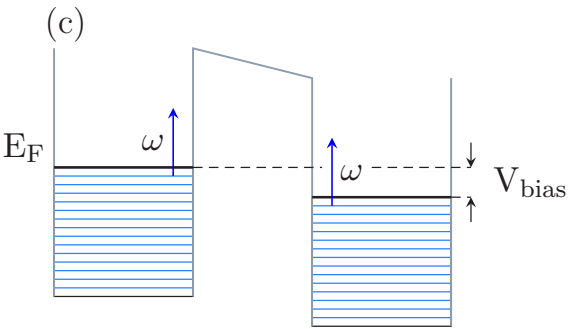

(e)

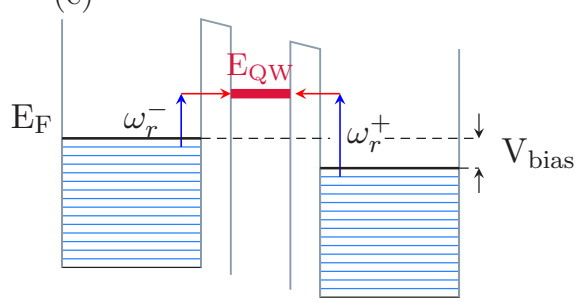

FIG. 1. (a) Geometry of the studied system. (b)-(e) Schematic electronic structure of the tunneling junction and the main optical absorption processes. The electronic levels (blue lines) of each slab are occupied up to the Fermi level $\left(E_{F}\right)$. The unoccupied electronic state of the QW $\left(E_{\mathrm{QW}}\right)$ is shown in red. When the bias $V_{\text {bias }}$ is applied, the $E_{F}$ levels of each slab experience a corresponding energy shift. Excited states can be accessed by photon absorption. Blue arrows indicate the intraslab photon absorption processes. Red arrows indicate the transitions involving QW and leading to PAT.

$11 \mathrm{~nm}$. The separation between the metal slabs is $d=0.8 \mathrm{~nm}$ as measured between the opposite metallic surfaces across the junction. The dielectric spacer layers between QW and slabs are modelled as vacuum layers $(\mathrm{V})$. The only constraints with respect to the separation between the metal slabs $d$ and the thickness of the spacer layers at the metal/QW interfaces are such that (i) the metal slabs are electronically decoupled in the absence of the QW and (ii) the QW-localized electronic state is strongly coupled to the metal slabs and can serve as an efficient intermediate for the electron transport across the junction (see below). In a different context, the multilayer metal-dielectric-metal structures attract a lot of interest as optical metamaterials or waveguide claddings [50-52] in the infrared and visible frequency ranges, as well as they are used for the XUV optics [53,54].

Prior to the discussion of the TDDFT results, let us outline some simple physical considerations behind the proposed strategy of the active control of the photon absorption. In the linear response regime, an efficient absorption occurs when the photon energy matches the energy difference between electronic states coupled by the time-dependent field. The electronic structure of the system is sketched in Fig. 1, together with the main optical absorption processes.

For the metallic slabs, the quantization of electron motion in $z$ direction results in discrete energies of electronic states $E_{j}, j=1,2, \ldots$ Each $z$-quantized state gives rise to the 2D continuum $E\left(j, k_{\|}\right)=E_{j}+k_{\|}^{2} / 2 m$, where $k_{\|}$is the electron momentum parallel to the surface and $m$ is the electron effective mass [48,49]. In the absence of the QWS in the junction, the metal slabs are quasidecoupled. The absorption of the $z$-polarized light is dominated by the intraslab transitions between occupied and empty electronic states at fixed $k_{\|}$. Note also that because of the planar symmetry, the induced fields vanish outside each slab. If a small dc bias voltage $\left(V_{\text {bias }}\right)$ is applied across the junction the entire electronic band corresponding to the given slab experiences a homogenous energy shift. In particular, the Fermi levels $E_{F}$ of the metal slabs across the junction are shifted by $V_{\text {bias }}$ one with respect to the other. Within each individual slab, the energy spacing between the electronic states is preserved and only the vacuum tails of their wave functions are affected. As a result, the absorption cross section is not sensitive to an applied bias in this case.

Consider now the sandwich metal-QW-metal nanostructure characterized by an unoccupied QWS at an energy $E_{\mathrm{QW}}$ above $E_{F}$ of metal slabs. Coupling of the QWS with the electronic states of the slabs opens the possibility of photon assisted resonant electron tunneling between the metal slabs [21-29]. The electron excitations involving the QWS lead to additional energy absorption channels with threshold frequency $\omega_{r}=$ $E_{\mathrm{QW}}-E_{F}$ [Fig. 1(d)]. If a small dc bias voltage $\left(V_{\text {bias }}\right)$ is symmetrically applied across the junction [Fig. 1(e)], the energy levels of the metal slabs are shifted by $\pm V_{\text {bias }} / 2$ with respect to the energy $E_{\mathrm{QW}}$ of the QWS located at the middle of the junction. Thus the photon assisted tunneling current across the junction and so the optical absorption will be modified. In particular, one would expect different threshold frequencies for the excitations from the slabs located to the left and to the right from the junction $\omega_{r}^{ \pm}=E_{\mathrm{QW}}-E_{F} \pm V_{\text {bias }} / 2$. Furthermore, one can use a gate electrode to control the QWS energy-level position, $E_{\mathrm{QW}}$.

To demonstrate the electrical control of the optical absorption in the system, we performed quantum-mechanical calculations within the TDDFT framework [55,56]. Based on a time-dependent Kohn-Sham (KS) scheme, the TDDFT allows one to follow the time evolution of the electronic density of the many-electron system in response to a time-dependent external field. The metal slabs are described with the jellium model (JM) [57], which has been extensively used to address quantum effects in plasmonic systems allowing semiquantitative 
predictions [58-60] confirmed experimentally and with $a b$ initio studies [12,61-67]. Within the JM, the atomic cores are not treated explicitly, but represented with a positive background charge of the density $n^{+}=\left(\frac{4 \pi}{3} r_{s}^{3}\right)^{-1}$. Here, the Wigner-Seitz radius $r_{s}=2.07 a_{0}\left(a_{0}=0.053 \AA\right)$ is used; it corresponds to the Al metal, a prototype free-electron metal whose valence electrons dynamics is well captured by the JM. Moreover, $\mathrm{Al}$ has recently attracted growing interest in the context of plasmonic applications [68-71].

For the case of $z$-polarized incident electromagnetic radiation, the electron density dynamics is described with 1D KS coupled equations:

$$
i \frac{\partial \phi_{j}(z, t)}{\partial t}=\left[-\frac{1}{2} \frac{d^{2}}{d z^{2}}+V_{\mathrm{eff}}(n, z, t)\right] \phi_{j}(z, t) .
$$

Initial conditions $\phi_{j}(z, t=0)$ are given by the occupied $(j=1, \ldots, N) \mathrm{KS}$ orbitals of the unperturbed ground-state system. Equations (1) are solved on a spatial mesh of points. The time-dependent electron density of the system $n(z, t)$ is given by $n(z, t)=\frac{1}{\pi} \sum_{j=1}^{N}\left(E_{F}-E_{j}\right)\left|\phi_{j}(z, t)\right|^{2}$. The spin degeneracy and the translational invariance of the problem are thus accounted for.

The effective one-electron potential is given by the sum of several terms:

$$
V_{\mathrm{eff}}(n, z, t)=V_{H}(n, z, t)+V_{\mathrm{xc}}(n, z, t)+V_{\mathrm{QW}}(z)+V_{\mathrm{ext}}(z, t) .
$$

The first term, $V_{H}$, is the Hartree potential obtained from the solution of the Poisson's equation. We thus neglect the retardation effects, which is a good approximation considering the small relevant size of the system. The second term, $V_{\mathrm{xc}}$, is the exchange-correlation potential of Gunnarson and Lundqvist [72] used within the adiabatic local density approximation (ALDA) [55]. The electron interaction with the QW structure is represented by the model potential $V_{\mathrm{QW}}(z)=$ $V_{0}\left[1+\exp \left(\frac{|z|-b}{\delta}\right)\right]^{-1}$, with $V_{0}=-3.5 \mathrm{eV}, b=2 a_{0}$, and $\delta=$ $0.25 a_{0}$. The above choice of the parameters allows one to introduce an unoccupied quantum-well localized electronic state between the vacuum level and the Fermi levels of the slabs. Finally, $V_{\text {ext }}(z, t)=z \mathcal{E}(t)$ describes the electron interaction with the external electromagnetic field. It can be given by one or several contributions corresponding to the optical and THz pulses, as well as to a dc field $E(t)=\mathcal{E}_{\text {bias }}$. Screening of the static field inside the Al slabs results in a homogeneous down/up energy shift of the electron energy levels of the left/right slabs. The situation is equivalent to a dc bias, $V_{\text {bias }}$, applied between the slabs with $V_{\text {bias }}=d \mathcal{E}_{\text {bias }}$.

In Fig. 2, we show the effective one-electron ground-state potential of the metal-QW-metal system $V_{H}+V_{\mathrm{xc}}+V_{\mathrm{QW}}$ [panel (a)], and the corresponding electronic structure [panel (b)]. The thickness of $\mathrm{Al}$ slabs is $L=5.5 \mathrm{~nm}$. The projected density of electronic states (PDOS) at the $\bar{\Gamma}$ point $\left(k_{\|}=0\right)$ has been obtained from the single active electron dynamics in the self-consistent KS ground-state potential. The wave packet propagation technique has been used as described elsewhere [73]. In the absence of the QW, the finite $z$-size effect results in a quantized energy level structure [blue line in Fig. 2(b)]. The
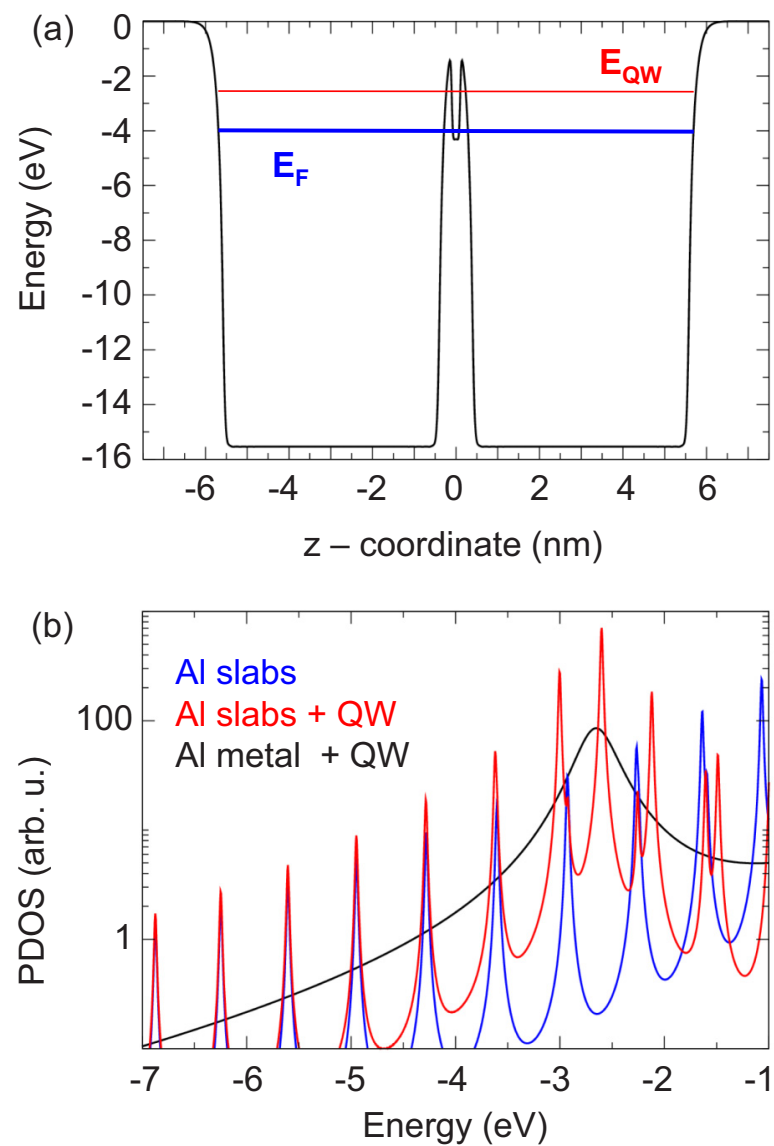

FIG. 2. (a) Ground-state potential of the metal-QW-metal nanostructure. Horizontal lines show the energies of the Fermi level of the metal slabs $E_{F}$ (blue) and QW-localized state $E_{\mathrm{QW}}$ (red). (b) Projected density of one-electron states at the $\bar{\Gamma}$ point $\left(k_{\|}=0\right)$ calculated with the wave packet propagation technique for different model systems: Al slabs of thickness $L=5.5 \mathrm{~nm}$ separated by a vacuum junction (blue line); Al slabs of thickness $L=5.5 \mathrm{~nm}$ separated by a junction functionalized with a QW structure (red line); QW structure between semi-infinite slabs (black line). Artificial broadening of $0.025 \mathrm{eV}$ has been introduced for the discrete states.

electronic states symmetric and antisymmetric with respect to $z=0$ are nearly degenerate because of the vanishing interaction between metal slabs. When the QW is introduced in the junction, the degeneracy is lifted in the vicinity of the QWS energy. While the antisymmetric states are affected only slightly, the symmetric states are shifted in energy because of the coupling with the QWS that emerges at $E_{\mathrm{QW}}=-2.6 \mathrm{eV}$ (throughout the paper, the energies of the electronic states are referred with respect to the vacuum level). The coupling of the QWS with the metal slabs opens an efficient channel for the PAT across the junction, where the QWS serves as a gateway [21-29]. In the case of the semi-infinite metal leads, the QWS broadens into the resonance with a width $\Gamma_{\mathrm{QW}}=0.4 \mathrm{eV}$, reflecting the fast decay (lifetime $\tau=1 / \Gamma_{\mathrm{QW}}=1.6 \mathrm{fs}$ ) of the QW electron population by resonant electron transfer into the metal leads. Note that at resonance the transmission probability of the excited electron between the leads can reach unity as has been widely discussed in the literature [24,74]. 

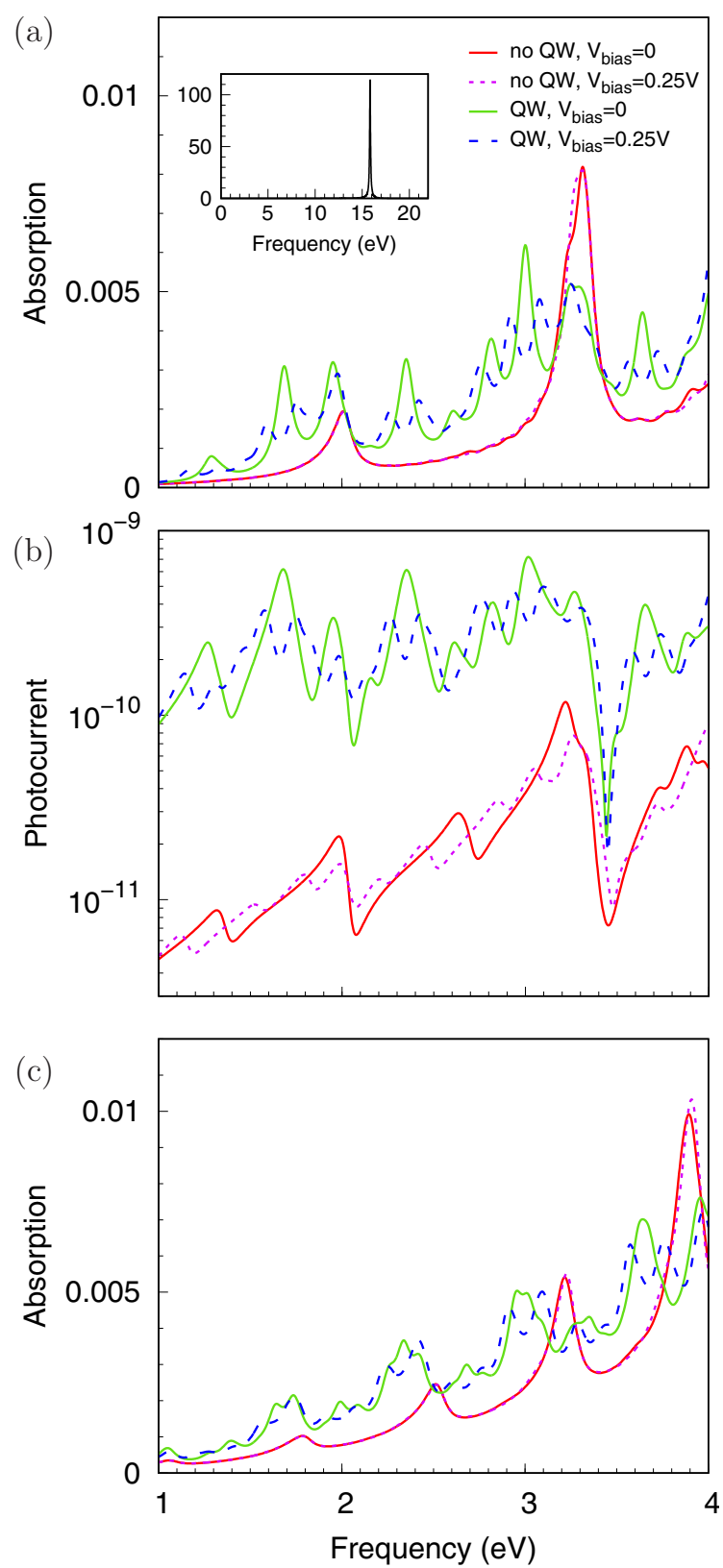

FIG. 3. (a) Optical absorption cross section per unit area calculated with TDDFT for bare (no QW) and QW functionalized $(\mathrm{QW})$ junctions without $\left(V_{\text {bias }}=0\right)$ and with $\left(V_{\text {bias }}=0.25 \mathrm{~V}\right)$ an applied bias. The thickness of Al slabs is $L=5.5 \mathrm{~nm}$. (Inset) Optical absorption for a broader energy range, comprising a plasmon mode excitation. (b) Spectrum of the photon assisted current across the junction between the $L=5.5 \mathrm{~nm} \mathrm{Al}$ slabs. The color code is the same as in (a). (c) The same as (a) but for the $L=11 \mathrm{~nm}$ thick Al slabs.

\section{RESULTS AND DISCUSSION}

From our TDDFT calculations, we obtain that introducing the QW structure in the gap between two Al slabs results in strong electrically controllable change of the absorption spectra in the optical frequency range. In Fig. 3(a), we show the absorption cross section $\sigma(\omega)$ per unit area calculated with TDDFT for the metal-vacuum-metal and metal-QW-metal junctions with and without an applied bias. The cases of Al slabs with thickness $L=5.5 \mathrm{~nm}$ and $L=11 \mathrm{~nm}$ have been addressed. The absorption cross section is obtained

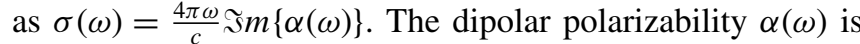
given by the time-to-frequency Fourier transform, $\alpha(\omega)=$ $\frac{1}{\mathcal{E}_{0}} \int p(t) e^{i(\omega+i \gamma / 2) t} d t$, of the time-dependent induced dipole per unit area, $p(t)$. The latter is calculated with TDDFT in response to the small impulsive perturbation $\mathcal{E}(t)=\mathcal{E}_{0} \delta(t)$. An artificial broadening of the dipole spectrum, $\gamma=0.1 \mathrm{eV}$, is introduced to take into account dissipative effects that are lacking in the TDDFT approach using the ALDA for exchange and correlation [75]. We note that for $z$-polarized incident electromagnetic field, the absorption spectra in all cases feature the main resonance at $\omega_{p}=15.8 \mathrm{eV}$ [see the insert of Fig. 3(a)] close to the bulk plasmon frequency of $\mathrm{Al}$ [76]. In what follows, we focus our attention on the lower photon energy range 1-4 eV relevant for optical applications.

In order to get a deeper insight into the photoassisted ac electron transport across the junction and its role in the optical absorption, we show in Fig. 3(b) the absolute value of the frequency-resolved current density calculated at the middle of the junction,

$$
J(\omega)=\int\left[J_{e}(t)-J_{0}(t)\right] e^{i(\omega+i \gamma / 2) t} d t .
$$

The current densities $J_{e}(t)$ and $J_{0}(t)$ correspond to the system excited (e) or not excited (0) by an impulsive field. Subtracting the latter allows one to remove the contribution of the applied bias and to focus on the optically excited currents.

In the case of the metal-vacuum-metal system (no QW), the width of the junction $d=0.8 \mathrm{~nm}$ is large enough so that the overlap of the electronic states across the vacuum gap is negligible and the ac tunneling currents are low [see Fig. 3(b)]. Since because of the charge neutrality the electric field of each slab vanishes at a distance of some $r_{s}$ outside its surfaces, the Al slabs are decoupled and respond independently to the optical excitation. This is further confirmed by performing the TDDFT calculations for the absorption cross section of the single Al slab in vacuum, $\sigma_{1}(\omega)$. We find that for the unbiased metal-vacuum-metal junction, $\sigma(\omega)=2 \sigma_{1}(\omega)$. In overall, the absorption cross section grows with $\omega$ (more electronic states below Fermi level are available for excitation). Intraslab electron-hole pair excitations [58] between $z$-quantized electronic states with the largest dipolar matrix elements result, for Al slabs of thickness $L=5.5 \mathrm{~nm}$, in the strong absorption features at 2.0 and $3.3 \mathrm{eV}$. Doubling the thickness of the slabs [ $L=11 \mathrm{~nm}$, Fig. 3(c)] leads to approximately twice denser spectrum of the $z$-quantized electronic states close to the Fermi level so that more absorption features are observed in Fig. 3(c). These features are less pronounced than for the thinner slab and the absorption spectrum appears smoother, as one would expect a discrete-to-continuum transition with increasing slab thickness. Since the absorption spectrum is dominated by the intraslab excitations, an applied bias of $0.25 \mathrm{~V}$ (applied dc field $E_{\text {bias }}=0.3 \mathrm{~V} / \mathrm{nm}$ ) has only a minor effect on the optical response in agreement with the qualitative discussion in Sec. II.

When the QW structure is introduced in the vacuum gap between metal surfaces, the optical response of the system is strongly modified. New resonances appear in the absorption spectrum in Fig. 3(a) owing to the dipole transitions which 
involve the QWS. The Fermi levels of the metal slabs in the ground state are located at $E_{F}=-3.85 \mathrm{eV}$ as calculated here. Therefore one expects that the onset of the optically induced transitions resonant with QWS will be at $\omega=E_{\mathrm{QW}}-E_{F}=$ $1.25 \mathrm{eV}$. This closely corresponds to the lowest QW-induced resonance in $\sigma(\omega)$ as seen in Fig. 3(a). For the thick slabs [Fig. 3(c)], the QW-induced absorption features appear broader and reflect contribution of several transitions with slightly different energies. Similar to the metal-vacuum-metal junction case, this reflects denser energy spectrum of the slab localized states, i.e., the evolution of the energy level structure from discrete to continuum. The role of the QWS as a gateway for efficient PAT tunneling between the leads can be inferred from the data in Fig. 3(b). Indeed, the QWS-induced absorption resonances are associated with strong increase in the tunneling current across the junction. Similar QWS-induced resonances in the coherent photon assisted tunneling in double-barrier systems with a gateway state are known in the $\mathrm{THz}$ frequency range for the semiconductor structures [21,22,27,34,77], or have been calculated in plasmonic gaps functionalized with a quantum dot structure $[35,38,39]$. As a general trend, owing to the (i) transitions involving the QWS, and (ii) overall decrease of the tunneling barrier, the photon assisted tunneling current is 1 to 2 orders of magnitude larger in the QW functionalized junction compared to the bare junction case.

Obviously, by changing the energy of the QWS with respect to the electronic structure of the metal films, one can modify the frequencies of the QWS-induced absorption peaks. An active electrical control of the optical response can be achieved by using a gate electrode or applying a bias voltage $V_{\text {bias }}$ across the QW junction as we demonstrate with TDDFT results in Fig. 3. It is noteworthy that an external bias results in a dc tunneling current between Al slabs. In a finite system, as considered here, the charge transfer across the gap would finally equilibrate the Fermi levels of the slabs, which in turn stop the tunneling current. To simulate the nonequilibrium process within the TDDFT framework, we then switch on the bias (field) progressively. The optical properties can be extracted during this transient regime where a quasi-steady-state current is temporarily established across the junction $[13,78]$.

For the symmetric case with $\pm V_{\text {bias }} / 2$ potential applied to the left/right $\mathrm{Al}$ slab, their electronic states experience the $\mp V_{\text {bias }} / 2$ energy shift. This is while the energy of the QWS is preserved giving rise to a modified absorption fingerprint. The resonant absorption features calculated for the bias-free case and ascribed to the excitations involving the QWS channel split into two peaks separated by $V_{\text {bias }}$. The higher/lower energy resonances correspond to the excitations into the QWS from the electronic states localized in the left/right slab. As a result, for an applied bias of $0.25 \mathrm{~V}$, we calculate about $50 \%$ variation of the optical absorption at the frequencies of the QWS-induced resonances for the $L=5.5 \mathrm{~nm}$ slabs. This variation is limited by the energy broadening of the absorption features due to the inelastic decay and dephasing mechanisms, which is taken as $0.1 \mathrm{eV}$ here $[79,80]$. For the thicker slabs, the broadening and smearing out of the absorption features because of the denser energy spectrum of the electronic states leads to the smaller bias induced variation $(\sim 20 \%$ at 2.3 and $3 \mathrm{eV}$ resonances). Thus, while qualitatively the possibility

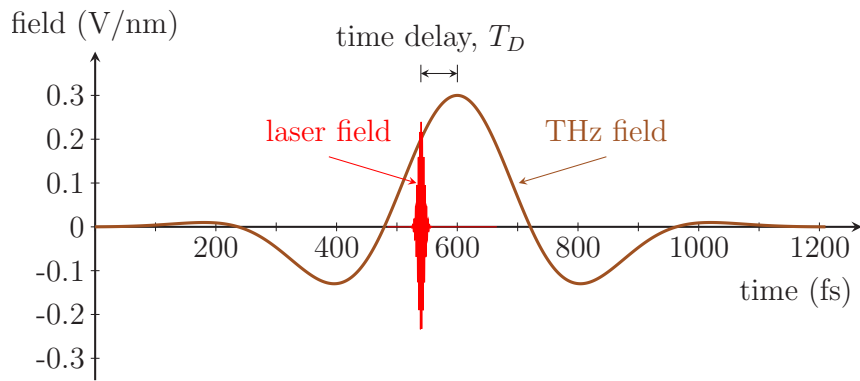

FIG. 4. Electric field of the THz and fs laser pulses as a function of time. The time delay between the pulses is explicitly shown.

of electrical control of the absorption persist for the thicker slabs, the thinner, $L=5.5 \mathrm{~nm}$, slabs correspond to better defined QWS induced absorption features and thus allow for stronger sensitivity to the applied bias. The quantum well confinement of the metallic electrons has a decisive influence on the results as far as the control strategy using QWS induced resonances is concerned. The $L=11 \mathrm{~nm}$ slab case represents an intermediate situation where the discrete spectrum of the energy levels evolves to the continuum of energy bands in thick metal. In this latter case, the absorption features due to the QWS will be smeared out and an applied bias will affect the onset of the transitions from the Fermi levels to the QW state, i.e., the frequency threshold for an increased optical absorption. At present, the computational constraints do not allow to reach the thick metal limit.

While above we demonstrated the electrical control of the optical properties of the metal-QW-metal structure using the dc applied bias, a different control strategy is addressed in what follows. We consider the $L=5.5 \mathrm{~nm} \mathrm{Al} \mathrm{slab} \mathrm{case} \mathrm{showing}$ larger sensitivity to an applied dc electric field. A "pump" $\mathrm{THz}$ pulse is used to create an unbalance between the Fermi levels of the Al slabs and a femtosecond laser pulse (fs) in the near IR-Vis frequency range is used to "probe" the transient absorption properties of the nanojunction. The electric fields of the pulses are taken as

$$
\mathcal{E}(t)=\mathcal{E}_{s} \exp \left[-\frac{\left(t-t_{s}\right)^{2}}{\tau_{s}^{2}}\right] \cos \left(\omega_{s}\left(t-t_{s}\right)\right),
$$

where $s=(\mathrm{THz}, \mathrm{fs})$ stands for the radiation source. We used $E_{\mathrm{THz}}=0.3 \mathrm{~V} / \mathrm{nm}, \omega_{\mathrm{THz}}=8.4 \mathrm{meV}\left(\operatorname{period} 2 \pi / \omega_{\mathrm{THz}}=484\right.$ fs), and $\tau_{\mathrm{THz}}=242 \mathrm{fs}$. The electric field of the laser pulse $\mathcal{E}_{\mathrm{fs}}=0.25 \mathrm{~V} / \mathrm{nm}$ is set such that the peak intensity of the laser is $10^{10} \mathrm{~W} / \mathrm{cm}^{2}$. Laser pulses with durations $\tau_{\mathrm{fs}}=7.3 \mathrm{fs}$ (frequency bandwidth $\Delta \omega=0.2 \mathrm{eV}$ ) and $\tau_{\mathrm{fs}}=24.2 \mathrm{fs}(\Delta \omega=$ $0.06 \mathrm{eV})$ are used. The electric field strengths are such that nonlinear polarization effects or multiphoton absorption processes can be neglected. Example of two laser pulses is shown in Fig. 4. The time delay $T_{D}=t_{\mathrm{fs}}-t_{\mathrm{THz}}$ between the pump and probe pulses determines the energy shift of the electron states localized in $\mathrm{Al}$ slabs induced by the $\mathrm{THz}$ field at the moment of the femtosecond (fs) pulse arrival. Because of its slow variation, the $\mathrm{THz}$ field is screened inside the Al slabs. It thus acts as an instantaneous dc field or instantaneous bias so that changing $T_{D}$ allows to actively tune the light induced electron dynamics and absorption of the energy of the fs laser pulse. 
To quantify the optical absorption in the nanojunction device, we calculated the energy $\Delta E$ transferred from the electromagnetic field of the fs laser pulse to the electronic system per unit surface area. Variation of the time delay $T_{D}$ and the frequency of the fs laser $\omega_{\mathrm{fs}}$ allows to explore different excitation regimes in quest for the active control. The TDDFT results are shown in Fig. 5 for fs laser pulse durations $\tau_{\mathrm{fs}}=24.2$ fs [upper row, (a)-(c)] and $\tau_{\mathrm{fs}}=7.3$ fs [lower row, (d)-(f)]. The 2D maps of $\Delta E$ variation as a function of $T_{D}$ and $\omega_{\mathrm{fs}}[(\mathrm{a}),(\mathrm{b}),(\mathrm{d})$, and (e)] provide qualitative insight into the excitation dynamics in the system. More quantitative assessment of the data can be obtained from the $\Delta E\left(T_{D}\right)$ dependence presented in Figs. 5(c) and 5(f) for several fixed frequencies of the fs laser close to QW-induced absorption resonances.

We start our analysis with the upper row of the panels in Fig. 5. The small frequency bandwidth of the fs laser pulse in this case provides high energy resolution. The pronounced time-delay dependence of the optical absorption can thus be observed. In the absence of the QW structure in the junction [Fig. 5(a)], the maximum electromagnetic energy absorption occurs for the fs laser frequency $\omega_{\mathrm{fs}}=\omega_{1}=1.95 \mathrm{eV}$. It corresponds to the absorption resonance due to the intraslab electron hole pair excitations found for the metal-vacuummetal structure in Fig. 3(a). The absorbed energy does not depend on the time delay between the pump and probe pulses, i.e., on the instantaneous value of the $\mathrm{THz}$ field at the moment of the fs pulse arrival. This result is analogous to the independence of the absorption spectra of the metalvacuum-metal structure on the applied bias (see Fig. 3).

The situation is very different for the QW functionalized junction [Fig. 5(b)]. As follows from the absorption spectra reported in Fig. 3 for the metal-QW-metal structure, in the frequency range studied here, the QWS induces an additional absorption resonance at $\omega_{\mathrm{fs}}=\omega_{2}=1.7 \mathrm{eV}$. A strong enhancement of the electromagnetic energy transfer to the electronic system at $\omega_{1}$ and $\omega_{2}$ frequencies is indeed found for large $T_{D}$ when the THz field is small. With decreasing $T_{D}$ and in sheer contrast with $\omega_{1}$ feature, the energy absorption in the QW-induced resonance frequency range shows a pronounced time-delay dependence. At small $T_{D}$, the instantaneous value of the $\mathrm{THz}$ field at the moment of the fs laser pulse arrival is large. The instantaneous bias $V_{\text {bias }}^{*}=d \mathcal{E}_{\mathrm{THz}}\left(t_{\mathrm{fs}}\right)$ associated with $\mathrm{THz}$ field splits the QWS induced absorption peak in higher and lower energy structures by analogy with results reported in Fig. 3. Thus an efficient energy absorption requires detuning of the fs laser frequency from the QW-induced resonance:

$$
\omega_{\mathrm{fs}}=\omega_{2} \pm d \mathcal{E}_{\mathrm{THz}}\left(t_{\mathrm{fs}}\right) / 2 .
$$

For the fixed frequency $\omega_{\mathrm{fs}}=1.7 \mathrm{eV}$, the loss of the resonance condition with absorption peak reduces the energy transfer by an order of magnitude [see Fig. 5(c)]. Notice that the weak $\mathrm{QW}$-induced feature at $1.3 \mathrm{eV}$ shows similar dependence on $T_{D}$ and $\omega_{\mathrm{fs}}$ as the $\omega_{2}$ resonance [see Fig. 5(b)]. Interestingly, from Eq. (5), it follows that the evolution of the frequency of the fs pulse corresponding to the maximum absorption reflects the temporal dependence of the $\mathrm{THz}$ field. In a way, this is similar to the streaking experiment in the attosecond laser physics, which allows one to probe the time evolution of a fs-IR laser pulse by measuring the energy spectrum of the electrons emitted by an XUV pulse [81,82].

Because of the finite lifetimes of the excited electrons in metals, the results obtained with narrow $\Delta \omega=0.06 \mathrm{eV}$ pulse bandwidth should be taken with caution. Indeed, for $\mathrm{Al}$, the inelastic decay rates $\Gamma_{i n}$ of the electronic excitations are in
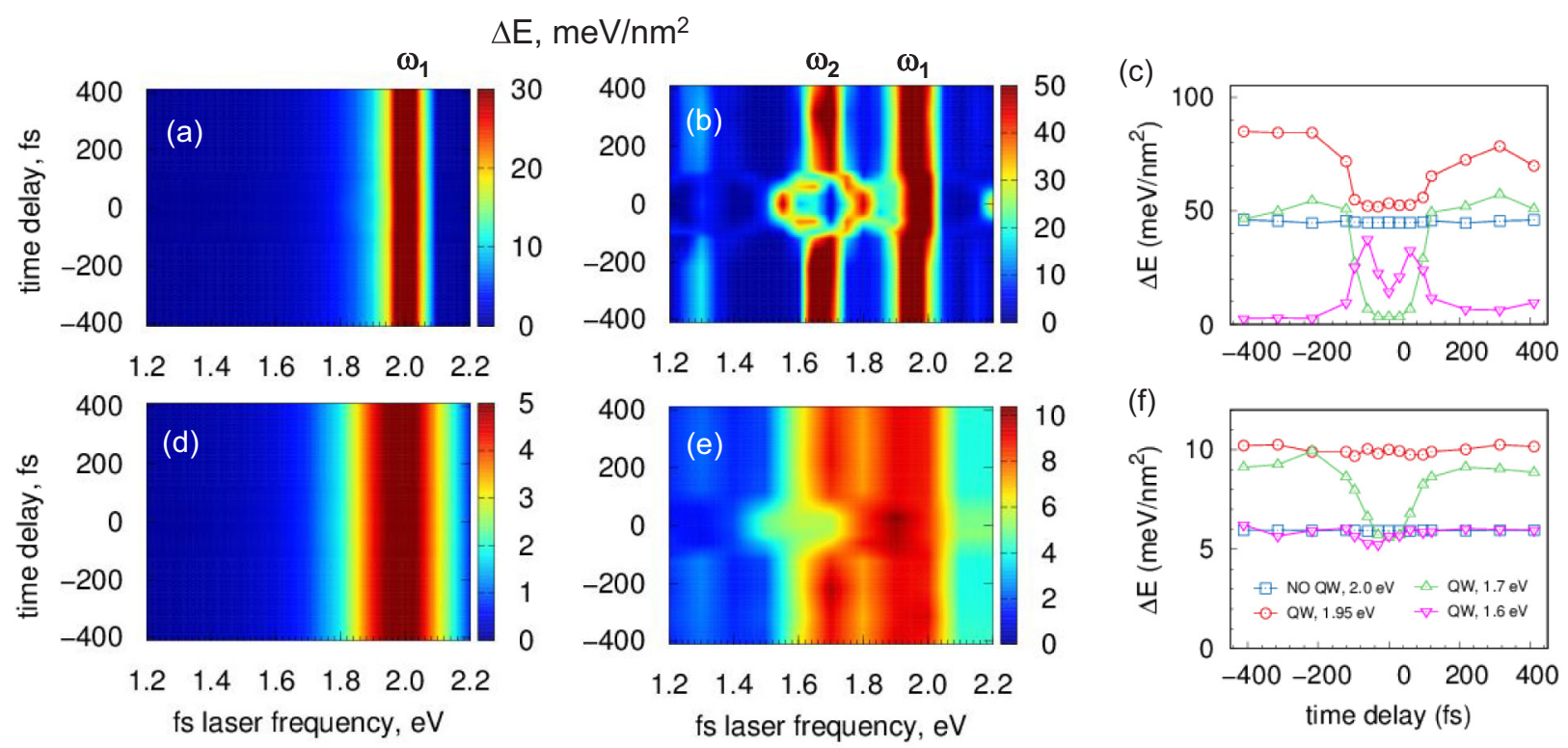

FIG. 5. Energy transfer from the fs laser pulse to electron excitations in the $L=5.5 \mathrm{~nm} \mathrm{Al} \mathrm{slab} \mathrm{case.} \mathrm{The} \mathrm{change} \mathrm{of} \mathrm{the} \mathrm{electron} \mathrm{energy}$ $\Delta E$ as a result of the interaction with the fs laser pulse, per unit surface area, is calculated with TDDFT. Results are shown for the fs laser pulse durations $\tau_{\mathrm{fs}}=24.2$ fs [upper row, (a)-(c)] and $\tau_{\mathrm{fs}}=7.3$ fs [lower row, (d)-(f)]. [(a), (b), (d), and (e)] 2D maps of $\Delta E$ as a function of the laser carrier frequency $\omega_{\mathrm{fs}}$ and of the time delay between the pump and probe pulses $T_{D}$. The results for metal-vacuum-metal [(a) and (d)] and metal-QW-metal [(b) and (e)] junctions are shown. [(c) and (f)] Cuts of the 2D maps showing $\Delta E$ dependence on the time delay $T_{D}$ for several $\omega_{\mathrm{fs}}$ fixed close to the main absorption features. 
the $60 \mathrm{meV}$ range for excitation energies around $2 \mathrm{eV}[79,80]$. That is, the energy uncertainty introduced by the multielectron processes, which is not accounted for in the present ALDA functional for exchange-correlation, is of the same order of magnitude as the fs pulse width. To check the robustness of the results with respect to energy broadening because of the inelastic processes, we performed calculations with short $\tau_{\mathrm{fs}}=7.3$ fs laser pulse with $\Delta \omega=0.2 \mathrm{eV}$ bandwidth. The $\Delta \omega$ in this case is much larger than $\Gamma_{i n}$ and it dominates the energy broadening effects. The corresponding results are shown in Figs. 5(d)-5(f). Essentially, the 2D maps of the absorbed energy $\Delta E$ are the energy convolutions of the 2D maps obtained with narrow laser pulse. The details of the variation of the energy deposited into the system are naturally smoothed in this case. However, the modulation of the absorbed energy with $T_{D}$ persists at the $\mathrm{QW}$-induced resonance and can be as high as $\sim 50 \%$. Therefore the possibility to control the optical absorption with $\mathrm{THz}$ fields is qualitatively robust.

\section{SUMMARY AND CONCLUSIONS}

In summary, we have studied the possibility of active electrical control of the optical response of a compound nanoscale device. In this device, a QW structure is sandwiched between two several-nanometer-thick metal slabs. Similar systems have been previously addressed, mainly with model one-electron approaches. Here, we use quantum TDDFT calculations allowing for the full account of the many-body effects such as the nonlocal response and dynamical screening. We have shown that an unoccupied QW state can serve as a gateway for the transport of excited electrons at optical frequencies. In turn, characteristic features in the absorption spectra emerge. Varying the energy of the QW state with respect to the energies of the electronic states of the metal slabs allows one to modify the absorption spectra. We have demonstrated that this opens the possibility for active electrical control of the absorption at optical frequencies using an applied $\mathrm{dc}$ bias or a $\mathrm{THz}$ field. In the latter case, the metal-quantumwell-metal device can be also used to trace the time evolution of the $\mathrm{THz}$ field.

The thin metallic slabs considered in this work possess quantized spectra of electronic states, which result in resonant structures in the optical absorption cross section. These absorption resonances have been used here for the active electrical control. The considered control strategy, however, should also hold for thick metallic films or plasmon gap nanoantennas [83-85]. In these cases, owing to the continuum of energy bands in a metal, the onset of transitions involving the QW state would appear as a frequency threshold for increased optical absorption. The dependence of the threshold frequency on the energy of the QW state with respect to the Fermi levels of the metals on both sides of the junction should be the key for the active electrical control strategy.

Our results show that $\mathrm{QW}$-functionalized junctions between metallic films can be utilized for the design of active devices allowing electrical control of the optical absorption. The actively controlled transfer of electromagnetic energy to electronic excitations and photoassisted currents has a wide range of possible applications such as photochemistry or solar energy harvesting.

\section{ACKNOWLEDGMENT}

A.K.K. acknowledges partial support by Spanish Ministerio de Economia y Competitividad (MINECO) through Project No. FIS2016-76617-P.

\section{APPENDIX}

In this Appendix, we give the details of the numerical implementation of the ALDA TDDFT calculations. The timedependent electron density of the nanostructure is represented by that of the KS noninteracting system, and it is given by the sum over the occupied one-dimensional KS orbitals $\phi_{j}(z, t)$,

$$
n(z, t)=\frac{1}{\pi} \sum_{j=1}^{N}\left(E_{F}-E_{j}\right)\left|\phi_{j}(z, t)\right|^{2},
$$

where $N$ is the total number of the occupied KS orbitals, and $E_{j}$ is the energy of the KS orbital $\phi_{j}(z, t=0)$ of the groundstate system. $N=62$ for the case of 5.5-nm-thick Al slabs and $N=124$ for the case of 11-nm-thick Al slabs. As discussed in the main text of the paper, because of the symmetry of the problem, the contribution of the electronic states involved in motion parallel to the surface can be factorized out and is accounted for by the $\frac{1}{\pi}\left(E_{F}-E_{j}\right)$ statistical weights (the spin degeneracy is also included). The KS orbitals evolve in time according to the nonlinear Schrödinger equations

$$
i \frac{\partial \phi_{j}(z, t)}{\partial t}=\left[\hat{T}+V_{\mathrm{eff}}(n, z, t)\right] \phi_{j}(z, t),
$$

where $\hat{T}=-\frac{1}{2} \frac{d^{2}}{d z^{2}}$ is the kinetic energy operator and $V_{\text {eff }}(n, z, t)$ is the effective one-electron potential.

With initial conditions $\phi_{j}(z, t=0)$ given by the KS orbitals of the ground-state system (no incident field), equations (A2) are numerically solved using the split-propagation technique [86-88]:

$$
\begin{gathered}
\phi_{j}(z, t+\Delta t)=\hat{U} \phi_{j}(z, t), \\
\hat{U}=e^{-i \frac{\Delta t}{2} V_{\text {eff }}\left(n, z, t+\frac{\Delta t}{2}\right)} e^{-i \Delta t \hat{T}} e^{-i \frac{\Delta t}{2} V_{\text {eff }}\left(n, z, t+\frac{\Delta t}{2}\right)} .
\end{gathered}
$$

Here, $\hat{U}$ is the evolution operator.

With Eqs. (A3) and (A4) discretized on the equidistant grid of $N_{z}$ knots in $z$ coordinate, $z_{l}=z_{0}+h(l-1), l=$ $1,2, \ldots, N_{z}$, the action of the $e^{-i \frac{\Delta t}{2} V_{\text {eff }}\left(n, z, t+\frac{\Delta t}{2}\right)}$ operator is local:

$$
e^{-i \frac{\Delta t}{2} V_{\text {eff }}\left(n, z, t+\frac{\Delta t}{2}\right)} \phi_{j}(z, t) \rightarrow e^{-i \frac{\Delta t}{2} V_{\text {eff }}^{l}} \phi_{j, l}(t) .
$$

Here, $V_{\text {eff }}^{l} \equiv V_{\text {eff }}\left(n, z_{l}, t+\frac{\Delta t}{2}\right)$ and $\phi_{j, l}(t) \equiv \phi_{j}\left(z_{l}, t\right)$.

The action of the nonlocal operator $e^{-i \Delta t \hat{T}}$ is calculated with the Fourier-grid pseudo-spectral method [87,88] using the discrete transforms based on the fast Fourier algorithm (FFT). Using the discrete Fourier transforms imposes the periodic boundary conditions on the $\mathrm{KS}$ orbitals at the boundaries of the grid. This is consistent with negligible electron emission in the present case,

$$
e^{-i \Delta t \hat{T}} \phi_{j}(z, t) \rightarrow \hat{F}^{-1} e^{-i \Delta t k^{2} / 2} \hat{F} \phi_{j, l}(t),
$$

where the discrete Fourier transform $(\hat{F})$ of the vector $\phi_{j, l}\left(l=1, \ldots, N_{z}\right)$ from the coordinate to the momentum $k$ representation is followed by the multiplication on the diagonal 
in momentum space matrix $e^{-i \Delta t k^{2} / 2} \delta_{k, k^{\prime}}$, and finally the discrete inverse Fourier transform $\left(\hat{F}^{-1}\right)$ from the momentum to coordinate representation is applied.

Since the electron density and thus $V_{\mathrm{eff}}\left(n, z_{l}, t+\frac{\Delta t}{2}\right)$ is not known at the half-time step, we use an iterative procedure. It consists in performing the first iteration with $V_{\text {eff }}\left(n, z_{l}, t+\right.$ $\left.\frac{\Delta t}{2}\right)=V_{\mathrm{eff}}\left(n, z_{l}, t\right)$ so that starting with $\phi_{j, l}(t)$, the estimation for $\phi_{j, l}(t+\Delta t)$ and thus for $n\left(z_{l}, t+\Delta t\right)$ and $V_{\text {eff }}\left(n, z_{l}, t+\right.$ $\Delta t)$ can be obtained. At the second iteration, we repeat the time step, and calculate $\phi_{j, l}(t+\Delta t)$ from $\phi_{j, l}(t)$ using $V_{\text {eff }}\left(n, z_{l}, t+\right.$ $\left.\frac{\Delta t}{2}\right)=0.5\left[V_{\mathrm{eff}}\left(n, z_{l}, t\right)+V_{\mathrm{eff}}\left(n, z_{l}, t+\Delta t\right)\right]$. This improves the prediction for the densities and potentials at $t+\Delta t$. Typically, three to four iteration loops are performed so that the time propagation scheme preserves the time reversal symmetry [89] and thus preserves the energy of the system without an external perturbation.

In our calculations, we use the time propagation step $\Delta t=$ 0.05 a.u. with full propagation time $\sim 1160$ fs. The coordinate meshes comprise $N_{z}=2048$ knots $\left(-270 \mathrm{a}_{0} \leqslant z \leqslant 270 \mathrm{a}_{0}\right)$ for the case of 5.5-nm-thick Al slabs and $N_{z}=4096$ knots $\left(-480 \mathrm{a}_{0} \leqslant z \leqslant 480 \mathrm{a}_{0}\right)$ for the case of 11-nm-thick Al slabs.

Several words are in order with respect to the groundstate calculation and the calculation of the Hartree potential $V_{H}(n, z, t)$. The latter is obtained as $V_{H}(n, z, t)=$ $-\left[v(z)+V_{D}(z)\right]$, where $v(z)$ solves the Poisson equation

$$
\frac{d^{2} v(z)}{d z^{2}}=-4 \pi\left[n^{+}(z)-n(z, t)\right]-\frac{d^{2} V_{D}(z)}{d z^{2}} .
$$

In Eq. (A7), $V_{D}(z)$ is the analytical potential of some dipole layer,

$$
V_{D}(z)=4 \pi D \frac{z}{2 \sqrt{z^{2}+a \exp \left(-z^{2} / b^{2}\right)}},
$$

where $a$ and $b$ are some parameters and $D=$ $\int\left(n^{+}(z)-n(z, t)\right) d z$ is the dipole moment of the system. Explicitly separating the potential difference at $z= \pm \infty$ because of the dipole layer allows to numerically solve Eq. (A7) for $v(z)$ with zero boundary conditions. Thus the pseudospectral method can be applied by representing $v(z)$ and the right-hand side (r.h.s.) of Eq. (A7) in the basis of $\sin (k z)$ functions and employing the discrete sine transforms.

The ground-state KS orbitals $\phi_{j}^{\mathrm{gs}}(z) \equiv \phi_{j}(z, t=0)$ [initial states for time propagation given by Eqs. (A3) and (A4)] are obtained performing the density functional theory calculations (DFT) [90]. The time-independent KS equations

$$
\left[\hat{T}+V_{H}+V_{\mathrm{xc}}+V_{\mathrm{QW}}\right] \phi_{j}^{\mathrm{gs}}=E_{j} \phi_{j}^{\mathrm{gs}}
$$

are solved on the discrete $z$ mesh by direct diagonalization of the Fourier grid Hamiltonian [91]. The $z$ mesh here is the same as the one used for solution of the time-dependent equations. An iterative procedure is applied allowing convergence of the system towards the ground state.
[1] A. Boardman, V. Grimalsky, Y. Kivshar, S. Koshevaya, M. Lapine, N. Litchinitser, V. Malnev, M. Noginov, Y. Rapoport, and V. Shalaev, Laser Photon. Rev. 5, 287 (2011).

[2] Q. Le-Van, X. Le Roux, A. Aassime, and A. Degiron, Nat. Commun. 7, 12017 (2016).

[3] Y.-W. Huang, H. W. H. Lee, R. Sokhoyan, R. A. Pala, K. Thyagarajan, S. Han, D. P. Tsai, and H. A. Atwater, Nano Lett. 16, 5319 (2016).

[4] Y. Yang, N. Kamaraju, S. Campione, S. Liu, J. L. Reno, M. B. Sinclair, R. P. Prasankumar, and I. Brener, ACS Photon. 4, 15 (2017).

[5] K. F. MacDonald, Z. L. Sámson1, M. I. Stockman, and N. I. Zheludev, Nat. Photon. 3, 55 (2009).

[6] H. W. Lee, G. Papadakis, S. P. Burgos, K. Chander, A. Kriesch, R. Pala, U. Peschel, and H. A. Atwater, Nano Lett. 14, 6463 (2014).

[7] X. Liu, J.-H. Kang, H. Yuan, J. Park, S. J. Kim, Y. Cui, H. Y. Hwang, and M. L. Brongersma, Nat. Nano. (to be published) (2017).

[8] A. Emboras, J. Niegemann, P. Ma, C. Haffner, A. Pedersen, M. Luisier, C. Hafner, T. Schimmel, and J. Leuthold, Nano Lett. 16, 709 (2016).

[9] C. Große, A. Kabakchiev, T. Lutz, R. Froidevaux, F. Schramm, M. Ruben, M. Etzkorn, U. Schlickum, K. Kuhnke, and K. Kern, Nano Lett. 14, 5693 (2014).

[10] R. A. Pala, K. T. Shimizu, N. A. Melosh, and M. L. Brongersma, Nano Lett. 8, 1506 (2008).

[11] S. Khatua, W.-S. Chang, P. Swanglap, J. Olson, and S. Link, Nano Lett. 11, 3797 (2011).
[12] D. Xiang, J. Wu, and R. Gordon, Nano Lett. 17, 2584 (2017).

[13] D. C. Marinica, M. Zapata, P. Nordlander, A. K. Kazansky, P. M. Echenique, J. Aizpurua, and A. G. Borisov, Sci. Adv. 1, e1501095 (2015).

[14] S. Thongrattanasiri, F. H. L. Koppens, and F. J. García de Abajo, Phys. Rev. Lett. 108, 047401 (2012).

[15] J. D. Cox and F. J. García de Abajo, Nat. Commun. 5, 5725 (2014).

[16] Z. Fei, A. S. Rodin, G. O. Andreev, W. Bao, A. S. McLeod, M. Wagner, L. M. Zhang, Z. Zhao, M. Thiemens, G. Dominguez, M. M. Fogler, A. H. Castro Neto, C. N. Lau, F. Keilmann, and D. N. Basov, Nature (London) 487, 82 (2012).

[17] A. N. Grigorenko, M. Polini, and K. S. Novoselov, Nat. Photon. 6, 749 (2012).

[18] F. H. L. Koppens, T. Mueller, P. Avouris, A. C. Ferrari, M. S. Vitiello, and M. Polini, Nat. Nanotechnol. 9, 780 (2014).

[19] P. K. Tien and J. P. Gordon, Phys. Rev. 129, 647 (1963).

[20] J. R. Tucker and M. J. Feldman, Rev. Mod. Phys. 57, 1055 (1985).

[21] M. Grifoni and P. Hänggi, Phys. Rep. 304, 229 (1998).

[22] G. Platero and R. Aguado, Phys. Rep. 395, 1 (2004).

[23] J. Iñarrea, G. Platero, and C. Tejedor, Phys. Rev. B 50, 4581 (1994).

[24] H. C. Liu, Phys. Rev. B 43, 12538 (1991).

[25] D. Sokolovski, Phys. Rev. B 37, 4201 (1988).

[26] M. H. Pedersen and M. Büttiker, Phys. Rev. B 58, 12993 (1998).

[27] A. Keller, O. Atabek, M. Ratner, and V. Mujica, J. Phys. B 35, 4981 (2002). 
[28] M. Galperin and A. Nitzan, Phys. Rev. Lett. 95, 206802 (2005).

[29] M. Galperin and A. Nitzan, Phys. Chem. Chem. Phys. 14, 9421 (2012).

[30] P. Hänggi, S. Kohler, J. Lehmann, and M. Strass, in Introducing Molecular Electronics, edited by G. Cuniberti, K. Richter, and G. Fagas (Springer, Berlin, Heidelberg, 2005), pp. 55-75.

[31] C. D. Lindstrom and X.-Y. Zhu, Chem. Rev. 106, 4281 (2006).

[32] A. Mayer, M. S. Chung, B. L. Weiss, N. M. Miskovsky, and P. H. Cutler, Nanotechnology 21, 145204 (2010).

[33] I. Urdaneta, A. Keller, O. Atabek, and V. Mujica, J. Chem. Phys. 127, 154110 (2007).

[34] J. K. Viljas and J. C. Cuevas, Phys. Rev. B 75, 075406 (2007).

[35] V. Kulkarni and A. Manjavacas, ACS Photon. 2, 987 (2015).

[36] U. Hohenester and C. Draxl, Phys. Rev. B 94, 165418 (2016).

[37] A. Thon, M. Merschdorf, W. Pfeiffer, T. Klamroth, P. Saalfrank, and D. Diesing, Appl. Phys. A 78, 189 (2004).

[38] P. Song, P. Nordlander, and S. Gao, J. Chem. Phys. 134, 074701 (2011).

[39] P. Song, S. Meng, P. Nordlander, and S. Gao, Phys. Rev. B 86, 121410 (2012).

[40] R. Baer, T. Seideman, S. Ilani, and D. Neuhauser, J. Chem. Phys. 120, 3387 (2004).

[41] P. Banerjee, D. Conklin, S. Nanayakkara, T.-H. Park, M. J. Therien, and D. A. Bonnell, ACS Nano 4, 1019 (2010).

[42] T. Wang and C. A. Nijhuis, Appl. Mater. Today 3, 73 (2016).

[43] F. Benz, C. Tserkezis, L. O. Herrmann, B. de Nijs, A. Sanders, D. O. Sigle, L. Pukenas, S. D. Evans, J. Aizpurua, and J. J. Baumberg, Nano Lett. 15, 669 (2015).

[44] R. M. Metzger, Chem. Rev. 115, 5056 (2015).

[45] J. Mertens, A. L. Eiden, D. O. Sigle, F. Huang, A. Lombardo, Z. Sun, R. S. Sundaram, A. Colli, C. Tserkezis, J. Aizpurua, S. Milana, A. C. Ferrari, and J. J. Baumberg, Nano Lett. 13, 5033 (2013).

[46] Y. Zhao, X. Li, Y. Du, G. Chen, Y. Qu, J. Jiang, and Y. Zhu, Nanoscale 6, 11112 (2014).

[47] T. Maurer, R. Nicolas, G. Lévêque, P. Subramanian, J. Proust, J. Béal, S. Schuermans, J.-P. Vilcot, Z. Herro, M. Kazan, J. Plain, R. Boukherroub, A. Akjouj, B. Djafari-Rouhani, P.-M. Adam, and S. Szunerits, Plasmonics 9, 507 (2014).

[48] T.-C. Chiang, Surf. Sci. Rep. 39, 181 (2000).

[49] P. Dowben, Surf. Sci. Rep. 40, 151 (2000).

[50] V. E. Babicheva, M. Y. Shalaginov, S. Ishii, A. Boltasseva, and A. V. Kildishev, Opt. Express 23, 31109 (2015).

[51] I. Avrutsky, I. Salakhutdinov, J. Elser, and V. Podolskiy, Phys. Rev. B 75, 241402 (2007).

[52] A. Husakou and J. Herrmann, Phys. Rev. Lett. 99, 127402 (2007).

[53] A. Mirone, M. Idir, P. Dhez, G. Soullie, and A. Erko, Opt. Commun. 111, 191 (1994).

[54] N. Kaiser and H. K. Pulker (eds.), Optical Interference Coatings, Springer Series in Optical Sciences Vol. 88 (Springer, Berlin, Heidelberg, 2003).

[55] M. A. L. Marques and E. K. U. Gross, Annu. Rev. Phys. Chem. 55, 427 (2004).
[56] R. E. Stratmann, G. E. Scuseria, and M. J. Frisch, J. Chem. Phys. 109, 8218 (1998).

[57] N. D. Lang and W. Kohn, Phys. Rev. B 3, 1215 (1970).

[58] E. Prodan, P. Nordlander, and N. J. Hallas, Chem. Phys. Lett. 368, 94 (2003).

[59] J. Zuloaga, E. Prodan, and P. Nordlander, Nano Lett. 9, 887 (2009).

[60] D. C. Marinica, A. K. Kazansky, P. Nordlander, J. Aizpurua, and A. G. Borisov, Nano Lett. 12, 1333 (2012).

[61] J. A. Scholl, A. García-Etxarri, A. L. Koh, and J. A. Dionne, Nano Lett. 13, 564 (2013).

[62] W. Zhu and K. B. Crozier, Nat. Commun. 5, 5228 (2014).

[63] K. J. Savage, M. M. Hawkeye, R. Esteban, A. G. Borisov, J. Aizpurua, and J. J. Baumberg, Nature 491, 574 (2012).

[64] H. Cha, J. H. Yoon, and S. Yoon, ACS Nano 8, 8554 (2014).

[65] H. Jung, H. Cha, D. Lee, and S. Yoon, ACS Nano 9, 12292 (2015).

[66] M. Barbry, P. Koval, F. Marchesin, R. Esteban, A. G. Borisov, J. Aizpurua, and D. Sánchez-Portal, Nano Lett. 15, 3410 (2015).

[67] A. Varas, P. García-González, J. Feist, F. J. García-Vidal, and A. Rubio, Nanophotonics (to be published) (2016).

[68] M. W. Knight, N. S. King, L. Liu, H. O. Everitt, P. Nordlander, and N. J. Halas, ACS Nano 8, 834 (2013).

[69] M. Castro-Lopez, D. Brinks, R. Sapienza, and N. F. van Hulst, Nano Lett. 11, 4674 (2011).

[70] G. Maidecchi, G. Gonella, R. Proietti Zaccaria, R. Moroni, L. Anghinolfi, A. Giglia, S. Nannarone, L. Mattera, H.-L. Dai, and M. Canepa, ACS Nano 7, 5834 (2013).

[71] B. Metzger, M. Hentschel, and H. Giessen, Nano Lett. 17, 1931 (2017).

[72] O. Gunnarsson and B. I. Lundqvist, Phys. Rev. B 13, 4274 (1976).

[73] E. V. Chulkov, A. G. Borisov, J. P. Gauyacq, D. Sánchez-Portal, V. M. Silkin, V. P. Zhukov, and P. M. Echenique, Chem. Rev. 106, 4160 (2006).

[74] D. Y. K. Ko and J. C. Inkson, Phys. Rev. B 38, 9945 (1988).

[75] C. A. Ullrich, J. Chem. Phys. 125, 234108 (2006).

[76] E. N. Economou, Phys. Rev. 182, 539 (1969).

[77] L. P. Kouwenhoven, S. Jauhar, K. McCormick, D. Dixon, P. L. McEuen, Y. V. Nazarov, N. C. van der Vaart, and C. T. Foxon, Phys. Rev. B 50, 2019 (1994).

[78] S. Kurth, in Fundamentals of Time-Dependent Density Functional Theory, edited by M. A. Marques, N. T. Maitra, F. M. Nogueira, E. Gross, and A. Rubio (Springer, Berlin, Heidelberg, 2012), pp. 337-350.

[79] I. Campillo, J. M. Pitarke, A. Rubio, E. Zarate, and P. M. Echenique, Phys. Rev. Lett. 83, 2230 (1999).

[80] V. P. Zhukov, E. V. Chulkov, and P. M. Echenique, Phys. Rev. B 72, 155109 (2005).

[81] F. Krausz and M. Ivanov, Rev. Mod. Phys. 81, 163 (2009).

[82] F. Krausz and M. I. Stockman, Nat. Photon. 8, 205 (2014).

[83] D. R. Ward, F. Huser, F. Pauly, J. C. Cuevas, and D. Natelson, Nat. Nanotechnol. 5, 732 (2010).

[84] A. Stolz, J. Berthelot, M.-M. Mennemanteuil, G. Colas des Francs, L. Markey, V. Meunier, and A. Bouhelier, Nano Lett. 14, 2330 (2014).

[85] J. Kern, R. Kullock, J. Prangsma, M. Emmerling, M. Kamp, and B. Hecht, Nat. Photon. 9, 582 (2015). 
[86] C. Leforestier, R. H. Bisseling, C. Cerjan, M. D. Feit, R. Friesner, A. Guldberg, A. Hammerich, W. Jolicard, G. Karrlein, H.-D. Meyer, N. Lipkin, O. Roncero, and R. Kosloff, J. Comput. Phys. 94, 59 (1991).

[87] M. D. Feit, J. A. Fleck, and A. Steiger, J. Comput. Phys. 47, 412 (1982).
[88] R. Kosloff, Annu. Rev. Phys. Chem. 45, 145 (1994).

[89] A. Castro, M. A. L. Marques, and A. Rubio, J. Chem. Phys. 121, 3425 (2004).

[90] W. Kohn and L. J. Sham, Phys. Rev. 140, A1133 (1965).

[91] C. C. Marston and G. G. Balint-Kurti, J. Chem. Phys. 91, 3571 (1989). 